\title{
EL PROYECTO EMECAM: DISCUSIÓN DE LOS RESULTADOS EN LAS CIUDADES PARTICIPANTES *
}

\begin{abstract}
Ferran Ballester Díez (1), Marc Sáez Zafra (2), Santiago Pérez-Hoyos (1) Antonio Daponte Codina (3), Juan B. Bellido Blasco (4), Álvaro Cañada Martínez (5), José M. ${ }^{a}$ Abad Díez (6), M. ${ }^{a}$ José Pérez Boillos (7), M. ${ }^{a}$ Eva Alonso Fustel (8), Margarita Taracido Trunk (9), Inés Aguinaga Ontoso (10), José Jesús Guillén Pérez (11), José M. ${ }^{a}$ Ordóñez Iriarte (12), Carme Saurina Canals (2) y José M.a Teuías Burillo (1) por el Grupo EMECAM.
\end{abstract}

(1) Institut Valencià d'Estudis en Salut Pública (IVESP). Dirección General de Salud Pública. Conselleria de Sanitat. Generalitat Valenciana.

(2) Universitat de Girona. Departament d'Economia.

(3) Escuela Andaluza de Salud Pública.

(4) Centro Salud Pública Área 02. Castelló. Conselleria de Sanitat.

(5) Dirección Regional de Salud Pública. Asturias.

(6) Dirección General de Salud Pública. Aragón.

(7) Departamento de Salud y Consumo. Ayuntamiento de Vitoria-Gasteiz.

(8) Departamento de Sanidad del Gobierno Vasco.

(9) Universidad de Santiago. Facultad de Medicina.

(10) Área de Sanidad y Medioambiente. Ayuntamiento de Pamplona.

(11) Centro Área Cartagena. Consejería de Sanidad de la Comunidad de Murcia.

(12) Dirección General de Salud Pública. Consejería de Sanidad y Scrvicios Sociales. Comunidad de Madrid.

(*) El proyecto EMECAM cuenta con una beca del Fondo de Investigaciones Sanitarias (Expediente núm 97/0051).

\section{RESUMEN}

En este artículo se comparan y discuten los resultados obtenidos en los análisis locales de las 14 ciudades participantes en el proyecto EMECAM. Se han analizado las series temporales de mortalidad, contaminantes (partículas en suspensión, $\mathrm{SO} 2, \mathrm{NO} 2$, $\mathrm{O} 3$ y $\mathrm{CO}$ ), temperatura y otros factores, obtenidos de registros de instituciones públicas. Utilizando regresión autorregresiva de Poisson, se ha estimado la relación a corto plazo entre la mortalidad y los indicadores de contaminación atmosférica en cada una de las siguientes ciudades: Barcelona, Gran Bilbao. Cartagena, Castellón. Gijón. Huelva, Madrid, Pamplona, Sevilla, Oviedo, Valencia, Vigo, Vitoria y Zaragoza. Los resultados indican que los valores de contaminación atmosférica en nuestro país son similares a los de otras ciudades europeas. Los niveles de los distintos contaminantes apuntan hacia el tráfico rodado como la fuente principal en la mayoría de ellas. Se encuentra una asociación entre la mortalidad y diferentes contaminantes en la mayoría de ciudades, aunque los resultados no son homogéneos entre las ciudades y presentan variabilidad en las distintas causas a estudio. En algunas ciudades, especialmente en aquellas de menor población. no se encuentran unos resultados que aporten evidencias de asociación, o bien los mismos son poco consistentes. El meta-análisis proporcionará estimaciones para el conjunto de las ciudades y permitirá una evaluación más clara del efecto retardado de la contaminación sobre la mortalidad. Es de destacar la participación como investigadores activos en este proyecto de técnicos de servicios de salud pública.

Palabras clave: Contaminación atmosférica. Mortalidad. Estudio multicéntrico. Series temporales.

Correspondencia:

Fcrran Ballester Dícz.

Institut Valencià d'Estudis en Salut Pública (IVESP).

Juan de Garay, 21.

46017 Valencia.

Teléfono 963869369

Fax 963869370

Correo electrónico: fballest@san.gva.es

\section{ABSTRACT}

\section{The EMECAM Project: A Discussion of the Findings in the Participating Cities}

This article draws a comparison and provides a discussion of the findings resulting from the local analyses of the 14 cities participating in the EMECAM Project. An analysis is made of the time series related to mortality, pollutants (particles in suspension, $\mathrm{SO} 2, \mathrm{NO} 2, \mathrm{O} 3$ and $\mathrm{CO}$ ), temperature and other factors taken from records of public institutions. By using Poisson autoregressive regression, an estimate has been made of the short-term relationship between the number of deaths and the air pollution indicators in each one of the following cities: Barcelona, Metropolitan Area of Bilbao, Cartagena, Castellón, Gi jón, Huelva, Madrid, Pamplona, Seville, Oviedo, Valencia, Vigo, Vitoria and Saragossa. The findings reveal the air pollution figures in our country to be similar to those of other European cities. The levels of the different pollutants point toward road traffic as being the main source of most of this pollution. A relationship has been found between the mortality and different pollutants in most cities, although the results are not homogeneous among the cities and show variability in the different causes under study. In some cities, especially in those having smaller populations, there have been no findings providing any evidence of a relationship, or the findings themselves are not highly consistent. The meta-analysis will provide estimates for all of the cities as a whole and will allow the possibility of making a more clear-cut assessment of the time lag impact of air pollution on the mortality. Worthy of special mention is the participation in this project of public health officiers as actively-involved researchers.

Key words: Air pollution. Mortality. Multicenter study. Time series. 


\section{INTRODUCCIÓN}

Los objetivos del proyecto EMECAM han sido presentados en otro artículo de este número'. Asimismo, para cada una de las ciudades que participan en el estudio, también se ha presentado la descripción de las variaciones diarias de la mortalidad, de los indicadores de contaminación atmosférica y la estimación de la asociación a corto plazo entre la mortalidad general y específica y la variación de los niveles de contaminación atmosférica. Además, con los resultados obtenidos tras la aplicación de una metodología estandarizada ${ }^{1,2}$ se realizará un meta-análisis cuyos resultados se publicarán más adelante.

En el presente trabajo nos ocuparemos fundamentalmente de comparar los datos de contaminación atmosférica entre las ciudades y discutir acerca de las estimaciones de la asociación a corto plazo entre las variaciones temporales de los contaminantes y la mortalidad, obtenidas en cada una de las ciudades ${ }^{3-15}$.

Por otro lado, el proyecto EMECAM también se planteaba, como objetivo para el conjunto del estudio multicéntrico, la elaboración y adaptación de un protocolo estandarizado, así como la aplicación de la metodología de análisis de series temporales. Estos aspectos merecen, igualmente, ser comentados.

\section{La medida de la exposición}

El proyecto EMECAM, como la mayoría de estudios epidemiológicos de los efectos de la contaminación atmosférica sobre la salud, utiliza datos de fuentes secundarias como indicadores de la exposición de la población a la misma. Es decir, se utiliza la información de las redes municipales o autonómicas de vigilancia de la contaminación, que miden valores de inmisión de los principales contaminantes en áreas urbanas y zonas industriales ${ }^{16}$. Las características de los sensores y los métodos analíticos utilizados responden a una normativa nacional y europea, y se integran en unos programas comunes de normalización y control de calidad. Su ubicación responde a criterios tales como la prioridad de la vigilancia de la contaminación emitida por centros industriales específicos o por las fuentes urbanas habituales (tráfico rodado).

En nuestro estudio, el interés en cuanto a la caracterización de la contaminación atmosférica se centra en valorar la exposición a la que se encuentra sometida la población de las ciudades participantes. Por todo ello, se estableció una serie de criterios para la obtención de los indicadores de contaminación que proporcionaran la medida más fiable de exposición en ambientes exteriores y, sobre todo, que reflejaran las variaciones diarias que se pudieran producir.

El número de captadores seleccionados varía según contaminante y ciudad, pero en ningún caso fue menor de 3 , estando todos ubicados en la zona urbana. Para un número importante de ciudades el número de captadores seleccionados fue superior a cinco, destacando el caso de Madrid, donde se utilizaron datos de 12 estaciones o más para cada uno de los contaminantes, excepto para el ozono, para el que únicamente se dispuso de datos de 3 estaciones captadoras ${ }^{12}$, y Zaragoza, donde se utilizaron 13 estaciones captadoras para los dos contaminantes analizados ${ }^{16}$. En los artículos correspondientes a cada una de las ciudades se puede obtener información más detallada en cuanto al número y localización de los captadores utilizados en cada caso. Es conveniente reseñar que en un número apreciable de estudios realizados en otros países se han utilizado los datos de pocas estaciones captadoras (a veces sólo una), o se cuenta con un número de captadores que varía a lo largo del periodo a estudio.

Otro aspecto destacable es la representatividad temporal de las series. Al tratarse de un diseño de series temporales, es funda- 
mental que, en el tiempo, la información de las estaciones captadoras sean representativa. Como se explica en otro trabajo de este númcro de la revista ${ }^{1}$, para el cálculo del promedio de cada contaminante se incluyeron sólo los valores de las estaciones captadoras que presentaron un porcentaje de datos válidos mayor al $75 \%$ de días para todo el periodo de estudio. Además, los valores perdidos se imputaron con los valores obtenidos tras ajustar una regresión lineal, utilizando los datos de las demás estaciones captadoras como variables explicativas. Como resultado de la aplicación de este método, el número de valores perdidos en el conjunto de las series de contaminación fue bajo. Únicamente en un caso, serie de los humos negros en Vigo, el porcentaje de datos válidos fue inferior al $95 \%(92 \%)$.

La tabla 1 muestra los estadísticos descriptivos de los niveles de contaminantes por ciudades. Sin olvidar las consideraciones expuestas más arriba (uso de datos secundarios, búsqueda de la representatividad temporal), es interesante examinar estos resultados de manera global y comparativa.
En conjunto, las ciudades estudiadas presentan patrones de contaminación diversos y abarcan un amplio abanico de niveles de los contaminantes analizados.

Todas las ciudades aportan datos de partículas expresadas bien como humos negros, bien como total de partículas en suspensión (PTS), o como partículas con un diámetro aerodinámico de 10 micras de mediana $\left(\mathrm{PM}_{10}\right)$. La ciudad que presenta los valores promedio más altos de humos negros es Vigo con $98,1 \mu \mathrm{g} / \mathrm{m}^{3}$, superior al registrado en Atenas $^{17}$, la ciudad con los niveles de humos negros más altos dentro del estudio $A P H E A$. Este valor representa prácticamente el doble de las concentraciones medias obtenidas en la mayor parte de las ciudades del proyecto $E M E C A M$, en el que los valores oscilaron entre los $40 \mathrm{y}$ $50 \mu \mathrm{g} / \mathrm{m}^{3}$. En Pamplona se encontraron unos niveles de humos negros considerablemente bajos $\left(9,7 \mu \mathrm{g} / \mathrm{m}^{3}\right)$, explicados a nivel local por el efecto de la construcción de la variante de la carretera que atravesaba la ciudad, por lo que se ha desviado todo el tráfico exterior.

Tabla 1

Descriptivos de los contaminantes* según ciudades en el proyecto EMECAM

\begin{tabular}{|lccccccccc|}
\hline \multicolumn{1}{|c}{ Ciudades } & Periodo & $\begin{array}{c}\text { Humos } \\
\text { negros 24h }\end{array}$ & PTS $24 h$ & $S_{2} 24 h$ & $S O_{2} I h$ & $N_{2} 24 h$ & $N O_{2} l h$ & CO 24h & $O_{3} 8 h$ max \\
\hline Barcelona & $91-95$ & $43,9(19,8)$ & $140,6(54,05)$ & $23,7(15,1)$ & $52,4(50,1)$ & $53,6(17,6)$ & $91,0(36,6)$ & $1,75(0,9)$ & $67,5(32,2)$ \\
Gran Bilbao & $92-96$ & $25,8(11,85)$ & $78,4(28,2)$ & $25,3(12,5)$ & $64,2(42,1)$ & $49,2(12,3)$ & $78,7(20,4)$ & - & - \\
Castellón & $91-95$ & $24,6(17,5)$ & - & $15,7(12,7) \dagger$ & - & - & - & - & - \\
Cartagena & - & - & $55,9(23,7)$ & $43,9(19,1)$ & $113,2(87,3)$ & - & - & - & - \\
Huelva & - & $42,5(15)^{* *}$ & & $11,7(7,6)$ & $59,1(70,6)$ & $32,9(10,9)$ & $72,7(27,8)$ & $0,67(0,4)$ & - \\
Sevilla & - & $45,1(14)^{* *}$ & - & $8,1(3,7)$ & $22,7(16,9)$ & $58,9(16,6)$ & $119,6(38,3)$ & - & - \\
Madrid & $92-95$ & $37,8(17,7)^{* *}$ & - & $35,5(27,2)$ & $77,9(64,5)$ & $71,0(20)$ & $123 \quad(44,6)$ & $2,10(1,2)$ & $42,1(27,8)$ \\
Pamplona & $91-95$ & $9,7(5,5)$ & - & $21,7(15,7) \dagger$ & - & - & - & - & - \\
Valencia & $94-96$ & $44,2(20,4)$ & - & $24,6(11,3)$ & $54,9(27)$ & $66,8(26,7)$ & $116,5(44,2)$ & $2,70(1,1)$ & $45,5(19,7)$ \\
Vigo & $91-94$ & $98,1(40,9)$ & - & $24,4(20,9) \dagger$ & - & - & - & - & - \\
Vitoria & $90-94$ & $51,2(30,2)$ & - & $18,04(10,3) \S$ & - & - & - & - & - \\
Gijón & $93-96$ & $52,0(28,6)$ & $82,9(31,1)$ & $34,1(22,8)$ & $87,8(57,1)$ & $45,1(17,9)$ & $77,9(22,5)$ & $1,90(0,9)$ & - \\
Oviedo & $93-96$ & $28,9(21,04)$ & $79,1(24,8)$ & $44,5(25,7)$ & $103,5(62,4)$ & $50,4(13,1)$ & $83,7(23,3)$ & $1,50(0,7)$ & - \\
Zaragoza & $91-95$ & $46,9(21,2)$ & - & $21,1(15,3) \dagger$ & - & - & - & - & - \\
\hline
\end{tabular}

* Descriptivos: Media (Desviación estándar) (en $\mu \mathrm{g} / \mathrm{m}^{3}$, excepto el CO en $\mathrm{mg} / \mathrm{m}^{3}$ ).

** PM10

$\dagger$ Método de la thorina (red manual).

$\S$ Cromatografia iónica (red manual). 
Las series de los valores diarios de humos negros presentan un patrón estacional, con valores más altos en los meses fríos que en los cálidos. Esta estacionalidad es más marcada en las ciudades del norte de España, posiblemente por el mayor uso de sistemas de calefacción de combustibles fósiles. Así, en Oviedo la razón entre la concentración del contaminante en el semestre frío y la concentración en los meses cálidos es de 2,3 , mientras que en otras ciudades (Barcelona y Valencia) es de 1,4.

Barcelona es la ciudad en la que se han registrado los niveles más altos de otro indicador de partículas, las PTS. Los $140 \mu \mathrm{g} / \mathrm{m}^{3}$ como promedio diario que se han alcanzado son comparables a los registrados en $\mathrm{Mr}$ lán ${ }^{18}$, los más altos registrados en la primera fase del proyecto APHEA. Sin embargo, los niveles moderados de humos negros observados en Barcelona, hacen pensar que la mayor parte de las PTS se deben a las partículas de mayor tamaño.

Las concentraciones de $\mathrm{PM}_{10}$ en Huelva, Sevilla y Madrid son del orden de las concentraciones de los humos negros en las otras ciudades. El patrón estacional, sin embargo, es diferente al de los humos negros, ya que en las dos ciudades andaluzas no hay diferencia en los niveles entre el semestre frío y el cálido. A este respecto, algunos autores argumentan que los niveles de humos negros y $\mathrm{PM}_{10}$ pueden considerarse equivalentes ${ }^{19}$, mientras que otros no consideran adecuada dicha comparación ${ }^{20}$. Los resultados de algunas investigaciones, como los obtenidos en el proyecto $P E A C E$, muestran que la relación entre humos negros y partículas en suspensión presenta una gran variabilidad de unos lugares a otros ${ }^{21}$.

Todas las ciudades disponen de promedios diarios de las concentraciones de $\mathrm{SO}_{2}$. Excepto en Vitoria, los datos de cinco de ellas proceden de redes manuales en las que el método de determinación analítica es el de la thorina. En esta ciudad, para la captación del contaminante se empleó $\mathrm{H}_{2} \mathrm{O}_{2}$ al
$0,3 \%$ y la determinación de la concentración del anión sulfato se realizó por cromatografía iónica con supresión química. El resto de las ciudades poseen datos que provienen de estaciones de muestreo automático en las que se utiliza el método de la fluorescencia ultravioleta. En general, los valores registrados son moderados o bajos. Oviedo y Cartagena son las ciudades con promedios más altos $\left(44,5 \mu \mathrm{g} / \mathrm{m}^{3}\right.$ y $43,9 \mu \mathrm{g} / \mathrm{m}^{3}$, respectivamente). Comparando los valores registrados en ambas ciudades según semestres ${ }^{1 l}$ se comprueba que la estacionalidad es mucho más marcada en Oviedo (razón concentración por semestres de 1,65), seguramente debido a que una fuente importante de contaminación es el uso de combustibles fósiles en las calefacciones. En Cartagena, sin embargo, los niveles entre semestres no muestran una variación importante (razón por semestres igual a 1,12), indicando una procedencia fundamentalmente industrial. En otras ciudades de la zona norte, como Zaragoza, se observa también un alto grado de estacionalidad en la concentración de $\mathrm{SO}_{2}$ (razón por semestres igual a 2,3). En las ciudades de Andalucía, Huelva y Sevilla, se registraron unos valores llamativamente bajos. Los promedios de las concentraciones de 1 hora de $\mathrm{SO}_{2}$ más elevados son los registrados en Cartagena y Oviedo. En algunas ciudades donde se dispone de datos de las redes automática y manual simultáneamente, la correlación entre ambas series ha sido baja. Por ejemplo, en Oviedo la media de la medición manual del $\mathrm{SO}_{2}$ fue de $25,4 \mu \mathrm{g} / \mathrm{m}^{3}$, casi la mitad que la automática.

En ocho de las ciudades se ha dispuesto de series de dióxido de nitrógeno. Entre ellas, Madrid es la que presenta los valores más altos, seguida de Valencia (promedios de 24 horas de $71 \mu \mathrm{g} / \mathrm{m}^{3}$ y $66,8 \mu \mathrm{g} / \mathrm{m}^{3}$ respectivamente). Estos valores son de una magnitud similar a la encontrada en las ciudades del proyecto $A P H E A$ con valores más altos, Atenas, Lyon y Londres ${ }^{22}$. Los valores más bajos se registraron en Huelva. Las concentraciones de $\mathrm{NO}_{2}$ no presentan diferencias remarcables por semestres. 
Los valores medios diarios de monóxido de carbono (CO) oscilaron entre $2,7 \mathrm{mg} / \mathrm{m}^{3}$ de Valencia y $0,7 \mathrm{mg} / \mathrm{m}^{3}$ en Huelva. Por último, se dispone de los promedios de las concentraciones de $\mathrm{O}_{3}$ de 8 horas en tres ciudades. Sus valores oscilan entre $42,1 \mu \mathrm{g} / \mathrm{m}^{3}$ en Madrid y $67,5 \mu \mathrm{g} / \mathrm{m}^{3}$ en Barcelona, niveles similares a los de otras ciudades europeas. Este contaminante presenta una estacionalidad distinta a las partículas y el $\mathrm{SO}_{2}$, con los niveles más altos en los meses cálidos (razón por semestres de 0,54 en Madrid y de 0,67 en Barcelona y Valencia).

En general, los valores registrados estarían dentro de los límites contemplados por la legislación vigente y las guías de calidad del aire de la $\mathrm{OMS}^{23}$, excepto en el caso de Vigo para los humos negros, donde se sobrepasan los $80 \mu \mathrm{g} / \mathrm{m}^{3}$ de promedio anual como valor límite ${ }^{24}$. Sin embargo, si tenemos en cuenta los valores previstos para las nuevas guías de calidad del aire de la $\mathrm{OMS}^{25}$ para el $\mathrm{NO}_{2}$, todas las ciudades de las que poseemos datos, excepto Huelva, excederían el valor límite propuesto $\left(40 \mu \mathrm{g} / \mathrm{m}^{3}\right)$.

\section{Asociación entre mortalidad y contaminación}

Todas las ciudades participantes en el proyecto $E M E C A M$ proporcionan en este número un avance de los resultados locales obtenidos. En algunos casos se ha optado por presentar una visión completa de las estimaciones de la asociación entre contaminantes y causas de muerte incluidas en el protocolo del estudio. En otros casos se exploran aspectos específicos, como la contaminación fotoquímica en el caso de Barcelona ${ }^{4}$ o la mortalidad por causas específicas, como en Zaragoza ${ }^{6}$. En el artículo sobre la ciudad de Vigo se abordan aspectos de avance metodológico; más en concreto, se realizan diferentes análisis incluyendo la regresión de Poisson utilizando modelos aditivos generalizados (GAM) .
Los resultados presentados corresponden a modelos con un solo contaminante. Se ha preferido utilizar este abordaje, en una primera instancia, para evitar problemas de colinealidad entre los contaminantes, dado que la contaminación es una mezcla compleja, los contaminantes comparten las mismas fuentes y presentan una correlación importante con los fenómenos meteorológicos. Se puede considerar que cada indicador de contaminación atmosférica incluye los efectos propios junto con los de los contaminantes emitidos con él o causados por él ${ }^{26}$. En análisis posteriores, con la orientación que proporcionan los resultados obtenidos hasta ahora en cada ciudad, se valorará la independencia de los efectos encontrados.

En general, los resultados obtenidos van en el sentido de la existencia de una asociación entre la contaminación atmosférica y la mortalidad en las ciudades incluidas en el proyecto. Sin embargo, los resultados no presentan homogeneidad en todos los casos. A continuación se comentan los más destacables para cada uno de los contaminantes, grupos de causas de mortalidad y períodos estudiados.

\section{Por contaminantes}

En este apartado, excepto en caso de mención expresa de algún grupo de causas específicas, nos referiremos a la mortalidad por todas las causas. Para las partículas se han encontrado riesgos relativos mayores de la unidad en la mayor parte de las ciudades. Para el grupo de todas las causas se ha encontrado una asociación estadísticamente significativa en el Gran Bilbao, en Barcelona (datos no presentados en el manuscrito) y en Valencia para todo el período, y en Huelva para el semestre frío. En otras ciudades, como Castellón, Pamplona o Vitoria, el escaso número de defunciones diarias puede haber dificultado la obtención de asociación significativa ${ }^{27}$. En algunos casos, la metodología empleada pucde no haber captado adecuadamente el efecto de la estacionalidad o 
de la temperatura. Es llamativo el caso de Vigo, en el que con el modelo no paramétrico se encuentra un efecto positivo en el retardo 1 , mientras que con la aproximación paramétrica se encontraba un efecto no esperado en el retardo 5. Por otro lado, la información sobre las variables meteorológicas en esta ciudad no reflejaba adecuadamente la exposición en la ciudad, ya que solo se pudo disponer de datos de la estación meteorológica del aeropuerto que está situado a 15 kilómetros de la ciudad y a mayor altitud. Ello provoca que las diferencias de temperatura entre la ciudad y el aeropuerto no sean constantes, sino que dependan de los meses del año. Debido a ello, el uso de modelos aditivos generalizados con control no paramétrico a partir de los datos de la serie, puede haber mejorado el control de la estacionalidad y de la temperatura y la humedad. Las asociaciones encontradas en general son del orden de las obtenidas en otros estudios, ya comentadas en otro trabajo de este número ${ }^{28}$.

En Sevilla y Madrid se presentan resultados con coeficientes negativos para el $\mathrm{PM}_{10}$. Aunque existe acuerdo en que dicho indicador es más adecuado que otros (como las PTS), para la evaluación y prevención de los efectos adversos en la salud, se recomienda establecer medidas de control de calidad que aseguren la fiabilidad en cuanto a las series de dichos contaminantes antes de utilizarlos en estudios epidemiológicos ${ }^{29}$. Por otro lado, estas dos ciudades se caracterizan por padecer unas temperaturas extremas en verano. En algunos estudios se ha encontrado una interacción entre los niveles de contaminación atmosférica y las temperaturas extremas ${ }^{30}$ sobre la mortalidad que será necesario controlar en posteriores análisis. Además, será preciso explorar más a fondo relaciones no lineales entre los niveles de contaminantes y la mortalidad.

Por otro lado, se debe hacer notar que resultados «no esperados» (RR menores de 1) se presentan habitualmente en retardos alejados, el cuarto o quinto. Algunos autores argumentan ${ }^{31}$ que cuanto más lejos se investiga el posible efecto retardado de la contaminación, menos seguros estamos de si la asociación que estimamos es debida al azar.

$\mathrm{E} 1 \mathrm{SO}_{2}$ es uno de los contaminantes sobre el que existe mayor controversia. En bastantes estudios realizados en los Estados Unidos no se ha encontrado una asociación clara de este contaminante con la mortalidad. Sin embargo, en los estudios europeos, fundamentalmente en el proyecto $A P H E A$ se informa de un efecto independiente del $\mathrm{SO}_{2}$. Los resultados de las ciudades del proyecto $E M E C A M$ que se presentan en este número no ofrecen una visión clara. Los retardos encontrados como de mejor ajuste son muy variables. El indicador del valor máximo horario parece ofrecer unos resultados más homogéneos en cuanto a los retardos y el sentido de las asociaciones, al menos en las ciudades de mayor tamaño, como Madrid, Barcelona (datos no presentados), Valencia y Bilbao. En cuanto al indicador del promedio diario se encontró una asociación significativa con la mortalidad en Madrid, Castellón y Huelva. En este caso la magnitud de la asociación es del orden de las encontradas en otros estudios. En el caso de Zaragoza se cncucntra una asociación positiva significativa con la mortalidad por causas circulatorias, más marcada en el semestre frío.

$\mathrm{El} \mathrm{NO} 2$ presentó, en general, un mejor ajuste en los retardos del día anterior y asociaciones positivas con una asociación significativa en el caso de Barcelona y para todas las causas. En Madrid, con el grupo de la mortalidad total, se encontró un RR menor de la unidad con el retardo 3 , aunque en los otros grupos de mortalidad estudiados se encontró una asociación significativa y positiva en el día simultáneo ${ }^{12}$. En el Gran Bilbao el riesgo relativo entre el $\mathrm{NO}_{2}$ diario (retardo 1) y la mortalidad por causas respiratorias se estimó en 1,048 $\left(\mathrm{IC}_{95 \%} 1,005-1,093\right)$ por cada $10 \mu \mathrm{g} / \mathrm{m}^{3}$.

En las tres ciudades en las que se estudia la asociación con el ozono se encuentra una asociación en sentido positivo, significativa 
al $95 \%$ en el caso de Barcelona y rozando esta significación en Madrid y Valencia. Estos resultados se sitúan en consonancia con los obtenidos en otros estudios ${ }^{22}$. A pesar de ello, este contaminante es de los más controvertidos a la hora de interpretar los resultados. Al ser un contaminante secundario, su concentración depende de la formación de otros contaminantes en combinación con otros factores, como la insolación, por lo que presenta una interacción con la temperatura $^{32}$. Como consecuencia de lo anterior, los niveles más altos se alcanzan en zonas periurbanas más que en el centro de las ciudades. Dos cuestiones más son las que afectan a la forma de la relación del ozono con la mortalidad (lineal, en forma de U, posible existencia de umbral) y una posible base fisiopatológica en cuanto al efecto retardado. En este sentido, aunque los resultados hallados hasta ahora scan de utilidad para orientarnos en los posibles efectos del ozono sobre la mortalidad son necesarios más estudios en profundidad.

Por último, la asociación con el monóxido de carbono resultó positiva en Madrid y Valencia, con significación estadística en esta última. En las dos ciudades asturianas, sin embargo, se encontró un RR menor que la unidad, y en Huelva no se encontró asociación. No hay que olvidar que cl CO debido a su estacionalidad se correlaciona altamente con otros contaminantes, fundamentalmente partículas y $\mathrm{SO}$, por lo que sería prematuro atribuirle el efecto encontrado sin haber descartado previamente la confusión por otros compuestos.

\section{Por grupos de mortalidad por edad y causas especificas}

No se observó, en general, una asociación de mayor magnitud en la mortalidad en mayores de 70 años con respecto a la mortalidad de la población general. Algunas de las variaciones en las estimaciones obtenidas son debidas principalmente a la selección de retardos diferentes. La inestabilidad en los coeficientes lleva a que la lectura de algunos resultados pueda ser engañosa o contradictoria si no se observa toda la información. Por ejemplo, en el caso de Madrid tanto las partículas como el $\mathrm{NO}_{2}$ presentaron una asociación negativa con la mortalidad por todas las causas en el retardo 4 y 3 respectivamente y, sin embargo, en relación a la mortalidad cardiovascular ambos contaminantes presentaron una asociación positiva y significativa en el día simultáneo (retardo 0). En Vitoria se detectó una mayor magnitud de la asociación de los humos negros en las personas mayores de 70 años.

En un número importante de ciudades la relación es más clara al analizar la mortalidad por causas del aparato circulatorio, como se ha encontrado en otros estudios ${ }^{19}$. Sin embargo, no se han obtenido unos resultados claros para la mortalidad por enfermedades respiratorias, quizás debido al bajo número de defunciones en algunas de las ciudades (debido a su pequeña población). Una explicación alternativa sería que, en algunas de las defunciones clasificadas como de causa circulatoria, el aparato respiratorio juegue un papel en el mecanismo fisiopatológico que desencadena la muerte que, sin embargo, no se refleja en el certificado de defunción.

En 7aragoza, ciudad en la que se ha estudiado la mortalidad por causas especificas dentro del grupo de enfermedades respiratorias $^{6}$, se ha encontrado una relación importante con las defunciones diarias por enfermedades del aparato respiratorio, especialmente para la enfermedad pulmonar obstructiva crónica (EPOC). La contaminación encontrada es más clara para la contaminación por humos y para los mayores de 70 años. El hecho de haberse estudiado en una sola ciudad y el escaso número de defunciones diaria para esta causa recomiendan tomar dichos resultados con precaución. 


\section{Por semestres}

En la mayoría de las ciudades, se encontró un efecto mayor en los meses cálidos, más clara en el caso de la contaminación por humos. Otros estudios realizados en Europa también han encontrado un efecto mayor de la contaminación en los meses cálidos ${ }^{33-38}$. Existen tres posibles explicaciones de este hallazgo. Por un lado, durante los meses cálidos otros determinantes de mortalidad, como las infecciones respiratorias, presentan menos influencia. Otra explicación, no incompatible con la anterior, podría relacionarse con el cambio en los hábitos de vida según las estaciones, en concreto el hecho de que la gente pasa más tiempo fuera de los edificios y que las ventanas permanecen abiertas de manera que la exposición a la contaminación atmosférica está más relacionada con la que se mide en los monitores. Por último, algunos estudios sugieren que existe una interacción entre contaminación atmosférica y temperaturas elevadas ${ }^{39}$, como se demostró en un estudio en Atenas $^{30}$. Se ha de hacer notar, por último, que en nuestro estudio tampoco existe un patrón similar en todas las ciudades en cuanto a este apartado ya que, por ejemplo, en Vitoria la asociación de la mortalidad con los humos negros fue mayor en los meses fríos.

\section{Consideraciones metodológicas}

Una de las consideraciones que deben de tenerse en cuenta a la hora de interpretar y discutir los resultados presentados en este número monográfico es que, a pesar de haberse obtenido mediante una técnica de análisis relativamente sofisticada, no están exentos de ciertas limitaciones.

En primer lugar, todos los resultados presentados corresponden a estimaciones realizadas con un solo contaminante en cada uno de los modelos. Esto significa que no podemos descartar el posible papel confusor de otros contaminantes. En la mayoría de las ocasiones este problema se ha abordado me- diante la introducción simultánea de dos contaminantes en el mismo modelo («modelos de dos contaminantes» $)^{40}$. A pesar de ello, sigue siendo posible que exista confusión residual por un tercer o cuarto contaminante. La construcción de modelos más complejos, aunque posible, viene limitada por la aparición de problemas de análisis de difícil resolución como la multicolineali$\operatorname{dad}^{41}$.

Otro punto a considerar es la medida de la exposición. Con este tipo de diseño se asume que los niveles de inmisión atmosférica son, en alguna medida, representativos de la exposición individual (al menos de su promedio) en la población analizada. Mediante estudios que valoraban simultáneamente la exposición individual y ambiental se ha comprobado que, aunque la concordancia entre los niveles detectados era relativamente baja ${ }^{42}$, sí que existe una buena correlación entre las variaciones temporales de ambas medidas $^{43}$. En línea con lo anterior, no hay que olvidar que se trata de estudios agregados, por lo que podríamos incurrir en un sesgo (falacia ecológica) al extrapolar los hallazgos encontrados de un nivel ecológico a un nivel individual. En el estudio $E M E C A M$ se asume que, en promedio, el total de la población está expuesto a los mismos niveles de contaminación. En epidemiología se reconoce que el grado de evidencia obtenido con este tipo de diseño no es tan alto como el que se derivaría de estudios de base individuales. Por esta razón, los resultados de estos estudios necesitan ser comprobados y confirmados con los resultados de estudios individuales.

Otro aspecto no menos importante se relaciona con la potencia de estos estudios para poder encontrar una asociación estadísticamente significativa. Como se ha comentado más arriba, en algunas ciudades, especialmente algunas de las de menor población, no se ha encontrado resultados que aporten evidencias significativas. Así, en Cartagena, Pamplona, Gijón y Oviedo, los resultados obtenidos hasta ahora no permi- 
ten concluir que existe una asociación detectable entre la contaminación y la mortalidad. En un artículo de reciente aparición del grupo de París que participa en el proyecto $A P H E A$, se dice que, para este tipo de estudios, es necesario poder estudiar un número de acontecimientos diarios (defunciones en este caso) «suficientemente» importante para esperar poner en evidencia una asociación estadísticamente significativa ${ }^{44}$. En nuestro estudio es destacable, es significativo el caso de Castellón, en el que se encuentra asociación de la mortalidad tanto de las partículas como con el $\mathrm{SO}_{2}$.

En cuanto a la metodología estadística utilizada conviene hacer algunos breves comentarios. La identificación de un modelo basal permite que, a la hora de establecer la relación entre la mortalidad y la contaminación, se hayan eliminado los posibles factores de confusión. Además, la inclusión de términos autorregresivos ayuda al control de la mala especificación del modelo (no haber incluido variables determinantes o no haber contemplado la forma funcional correcta). Pero, por otra parte, este control de variables puede llevar a una sobreparametrización que desestabilice las estimaciones del modelo.

En otros estudios se ha utilizado un modelo de regresión gaussiana a la hora de identificar el modelo basal ${ }^{44}$. El uso del modelo de Poisson autorregresivo desde el inicio ha permitido efectuar el ajuste en ciudades o causas donde el número de defunciones era relativamente bajo.

La elección de la posible forma funcional de la relación de algunas variables, como la temperatura, la tendencia, la estacionalidad y la humedad, pudiera ser cuestionable. Así, como se ha visto en Vigo, el uso de modelos aditivos generalizados podría controlar mejor estas variables de confusión. Sin embargo, a la hora de empezar la investigación, ni la metodología ni el software disponible estaban suficientemente extendidos, lo que di- ficultaba la progresión del proyecto y que pueda formar parte de posteriores análisis.

Por último, desde el punto de vista cuantitativo, la magnitud de las asociaciones encontradas es baja (riesgos menores de 1,5), por ello no se puede excluir definitivamente la posibilidad de que la asociación hallada sea debida a algún factor de confusión no tenido en cuenta o no adecuadamente controlado. Sin embargo, la consistencia de los resultados en múltiples estudios realizados en ciudades de todo el mundo apoya la evidencia de que la asociación encontrada no se debe al azar.

\section{Experiencia de trabajo multicéntrico}

Como se comentó al principio, además del objetivo central de evaluar en España el impacto a corto plazo de la contaminación atmosférica urbana sobre la mortalidad, el proyecto EMECAM contempla otros objetivos relacionados con la propia dinámica del proyecto. Entre ellos, destacaríamos tres:

En primer lugar, el desarrollo de una experiencia de trabajo multicéntrico. En este proyecto colaboran técnicos de servicios de salud pública, con investigadores de universidades y profesionales de escuelas de salud pública. En este sentido, la experiencia se considera enriquecedora para todos, tanto por el intercambio de conocimientos como por la posibilidad de obtener experiencia contrastada. Sin embargo, la propia marcha del proyecto ha evidenciado el escaso contacto que existe, en general, entre los profesionales responsables de las redes de vigilancia de la contaminación y los epidemiólogos. En ocasiones, las redes están más diseñadas para detectar alertas que para obtener una idea aceptable de los niveles de inmisión de la población.

El segundo se refiere a la posibilidad de familiarizarse con unas técnicas de análisis de datos epidemiológicos, en concreto metodología de series temporales, que hasta ahora era prácticamente desconocida en 
muchos de los grupos participantes. Ello ha permitido que cada uno de ellos adquiera las habilidades necesarias para realizar el análisis de los datos locales. De ese modo, la técnica empleada puede ahora ser utilizada en otros problemas de salud pública, como por ejemplo la vigilancia epidemiológica o la investigación de servicios de salud.

El tercer y último aspccto que se debe mencionar tiene relación con la actualización en nuestro país del estudio de los efectos de la contaminación atmosférica, en particular, y de los riesgos ambientales, en general. El desarrollo de proyectos como el $E M E C A M$, junto a otras iniciativas, puede ayudar a que en nuestro país se avance en el sentido de contribuir desde la epidemiología y la salud publica a la resolución de problemas relacionados con los riesgos ambientales.

A modo de conclusión, por un lado, los resultados presentados indican que los valores de contaminación atmosférica en nuestro país son similares a los de otras ciudades europeas. Los niveles de los distintos contaminantes indican que la fuente principal en la mayoría de ellas es el tráfico rodado. Por otro lado, se encuentra una asociación entre la mortalidad y diferentes contaminantes en la mayoría de las ciudades. Sin embargo, los resultados no son homogéneos entre las ciudades y presentan variabilidad en las distintas causas a estudio. En algunas de ellas, especialmente en aquellas de menor población, no se encuentran unos resultados que aporten evidencias de asociación, o bien son poco consistentes. El meta-análisis proporcionará estimaciones para el conjunto de las ciudades y debe permitir una evaluación más clara del efecto retardado de la contaminación sobre la mortalidad. Por último, se ha de destacar la participación como investigadores activos, en un proyecto especialmente complejo como el $E M E C A M$, de técnicos de servicios de salud pública.

\section{AGRADECIMIENTOS}

A los técnicos y responsables de las $\mathrm{Re}$ des de Vigilancia de la Contaminación Atmosférica, de los Registros de Mortalidad, de los Servicios de Vigilancia Epidemiológica y de los Centros Meteorológicos de cada una de las ciudades del estudio. Sin su trabajo este proyecto no se hubiera podido realizar. Sin embargo, en nuestra opinión, lo más importante es que con su dedicación contribuyen a la mejora de la calidad de vida y la salud de los ciudadanos.

$\mathrm{El}$ grupo $E M E C A M$ lo forman: $\mathrm{F}$ Ballester, S Pérez-Hoyos, JM Tenías, R Molina, J González-Aracil (Valencia, Centro coordinador); M Saez, MA Barceló, C Saurina, A Tobias (Barcelona); E. Alonso, K. Cambra (Bilbao); M Taracido, JM Barros, I Castro, A Figueiras, A Montes, E Smyth (Vigo); JM Ordóñez, E Aranguez, I Galán, AM Gandarillas (Madrid); I Aguinaga, MY Floristan, F Guillén, MS Laborda, MA Martínez, MT Martínez, PJ Oviedo (Pamplona); A Daponte, $R$ Garrido de la Sierra, JL Gurucelain, P. Gutiérrez, JA Maldonado, JL Martín, JM Mayoral, R Ocaña, J Serrano (Granada); JB Bellido, A Arnedo, F González (Castellón); JJ Guillén, Ll Cirera, L García, E Jiménez, MJ Martínez, S Moreno, C Navarro (Cartagena); MJ Pérez, A Alonso, JJ Estíbalez, MA García-Calabuig, (Vitoria); A Cañada, C Fernández, F Fernández, V García, I Huerta, V Rodríguez (Asturias); F Arribas, M Navarro, C Martos, MJ Rabanaque, E Muniesa, JM Abad, JI Urraca (Zaragoza); y J Sunyer como asesor del proyecto.

\section{BIBLIOGRAFÍA}

1. Ballester F, Sáez M, Alonso E, Taracido M, Ordóñez JM, Aguinaga $I$ et al. El proyecto EMECAM: Estudio multicéntrico español sobre la relación entre la contaminación atmosférica y la mortalidad. Antecedentes, participantes, objetivos, metodología. Rev Esp Salud Pública 1999; 73:163-175. 
2. Pérez-Hoyos S, Sáez M, Barceló M, Saurina C, Barceló MA, Ballester F. Protocolo EMECAM: Análisis del efecto a corto plazo de la contaminación atmosférica sobre la mortalidad. Rev Esp Salud Pública 1999; 73:177-185.

3. Guillén JJ, Cirera L, García-Marcos L, Navarro C, Jiménez E, Barber X et al. Mortalidad y contaminación por $\mathrm{SO}_{2}$ y partículas en Cartagena, 1992-1996. Proyecto EMECAM. Rev Esp Salud Pública 1999; 215-223.

4. Saurina C, Barceló M, Sáez M, Tobias A. Contaminación foto-química y mortalidad en la ciudad de Barcelona, 1991-1995. Rev Esp Salud Pública 1999; 199-207.

5. Taracido M, Figueiras A, Castro I. Efecto de la contaminación atmosférica sobre la mortalidad por todas las causas en la ciudad de Vigo (1991-1994). Rev Esp Salud Pública 1999; 275-281.

6. Arribas F, Alcalá T, Abad JM, Navarro M, Martos MC, Rabanaque MJ et al. Contaminación atmosférica y mortalidad por enfermedades respiratorias en Zaragoza (1991-1995). Rev Esp Salud Pública 1999; 293-301.

7. Pérez-Boíllos M, Alonso A, Estibalez J, García-Calabuig M. Efectos a corto plazo de la contaminación atmosférica sobre la mortalidad: Resultados del proyecto EMECAM en Vitoria-Gasteiz, 1990-94. Rev Esp Salud Pública 1999; 283-291.

8. Bellido J, Felis C, Arnedo A, González-Morán F, Herrero C, Safont L. Contaminación atmosférica y mortalidad en Castellón. Rev Esp Salud Pública 1999; 225-231.

9. Aguinaga I, Guillen F, Oviedo P, Floristan MY, Laborda MS, Martínez-Ramírez et al. Mortalidad diaria y contaminación atmosférica en la ciudad de Pamplona (1991-95). Rev Esp Salud Pública 1999; 253-257.

10. Tenías J, Pérez-Hoyos S, Molina R, González-Aracil J, Ballester F. Asociación a corto plazo entre contaminación atmosférica y mortalidad en la ciudad de Valencia. Resultados del Proyecto EMECAM. Rev Esp Salud Pública 1999; 267-273.

11. Cañada A, García-González J, Rodríguez-Suárez $\mathrm{V}$, Fernández-Noval $\mathrm{F}$, Fernández-Rodríguez $\mathrm{C}$, Huerta I. Contaminación atmosférica y mortalidad a corto plazo en 2 ciudades de Asturias. Rev Esp Salud Pública 1999; 187-197.

12. Galán I, Aránguez E, Gandarillas A, Ordóñez J, Aragonés N. Asociación entre la contaminación atmosférica y la mortalidad diaria en el municipio de Madrid, 1992-1995. Rev Esp Salud Pública 1999; 243-251.
13. Cambra K, Alonso E. Mortalidad diaria y contaminación atmosférica. Resultados del Gran Bilbao en proyecto EMECAM. Rev Esp Salud Pública $1999 ; 209-213$.

14. Ocaña R, Daponte A, Gutiérrez-Cuadra P, Mayoral JM, Gurucelain JL, Maldonado JA et al. Contaminación atmosférica y mortalidad en la ciudad de Sevilla, 1992-1996. Rev Esp Salud Pública 1999; 259-265.

15. Daponte A, Gutiérrez-Cuadra P, Ocaña R, Gurucelain J, Maldonado JA, Garrido R et al. Asociación entre la contaminación atmosférica y la mortalidad diaria en la ciudad de Huelva, 1993-1996. Rev Esp Salud Pública 1999; 233-241.

16. Aránguez E, Ordóñez J, Serrano J, Aragonés N, Fernández-Patier R, Gandarillas A et al. Contaminantes atmosféricos y su vigilancia. Rev Esp Salud Pública 1999; 73:123-131.

17. Touloumi G, Samoli E, Katsouyanni K. Daily mortality and «winter type» air pollution in Athens, Greece-a time series analysis within the APHEA project. J Epidemiol Commununity Health 1996; 50 Suppl 1:s47-51.

18. Vigotti M, Rossi G, Bisanti L, Zanobetti A, Schwartz J. Short term effects of urban air pollution on respiratory health in Milan, Italy, 1980-89. J Epidemiol Community Health 1996; 50 Suppl 1:s71-5.

19. Dockery D, Pope C. Acute respiratory effects of particulate air pollution. Annu Rev Public Health $1994 ; 15: 107-32$.

20. Moolgavkar S, Luebeck E. A critical review of the evidence on particulate air pollution and mortality. Epidemiol 1996; 7:420-8.

21. Roemer W, Hoek G, Brunekreef B, Schouten J, Baldini G, Clench-Aas $J$ et al. Effect of short-term changes in urban air pollution on the respiratory health of children with chronic respiratory symptoms - The PEACE project: Introduction. Eur Respir Rev 1998; 8:4-11.

22. Touloumi G, Katsouyanni K, Zmirou D, Schwartz J, Spix C, Ponce de Leon A et al. Short-term Effects of Ambient Oxidant Exposure on Mortality: A Combined Analysis within the APHEA Project. Am J Epidemiol 1997; 146:177-85.

23. World Health Organization (WHO) Regional Office for Europe. Air Quality Guidelines for Europe. WHO editor. Regional Publications European Series n. ${ }^{\circ} 23$. Copenhagen: World Health Organization; 1987. 
24. Boletín Oficial del Estado. Real Decreto 1321/92, por el que se modifica el RD $1613 / 85$ y se establecen nuevas normas de calidad del aire en lo referente a contaminación por dióxido de azufre y partículas en suspensión. BOE núm 289 de $30 / 10 / 1992$.

25. Leewen F. WHO Air Quality Guidelines for Europe. European Epi Marker 1997;

26. Medina S, Dab W, Quénel P, Ferry R, Festy B. Urban air pollution is still a public health problem in Paris. World Health Forum 1996;17:187-93.

27. Sunyer J. El estudio EMECAM sobre los efectos de la contaminación atmosférica. Rev Esp Salud Pública 1999; 73: 105-107.

28. Tenías J, Ballester F, Medina S, Daponte A. Estudios sobre los efectos de la contaminación atmosférica. Revisión de los trabajos originales que analizan su impacto en la mortalidad, 1994-1998. Rev Esp 1999; 145-163.

29. WHO. Measurement of particulate matter (PM10 and PM2.5) in Europe. Copenhague: WHO (Regional Office for Europe) 1998; (EUR/HFA target 21).p.1-14.

30. Katsouyanni K, Pantazopoulou A, Touloumi G, Tselepidaki I, Mousstris K, Asimakopoulos D, Poulopoulou G, Trichopoulos D. Evidence for interaction between air pollution and high temperature in the causation of excess mortality. Arch Environ Health 1993;48 (4):235-42.

31. Schwartz J, Spix C, Touloumi G, Bacharova L, Barumandzadeh $\mathrm{T}$, Le Tertre A, Pickarksi $\mathrm{T}$, Ponce de Leon A, Ponka A, Rossi G, et al. Methodological issues in studies of air pollution and daily counts of deaths or hospital admissions. J Epidemiol Community Health 1996;50 Suppl $1: \mathrm{S} 3-\mathrm{S} 11$

32. Sartor F, Snacken R, Demuth C, Walckiers D. Temperature, ambient ozone levels, and mortality during summer 1994, in Belgium. Environ Res $1995 ; 70(2): 105-13$.

33. Touloumi G, Pocock S, Katsouyanni K, Trichopoulos D. Short-term effects of air pollution on daily mortality in Athens: a time-series analysis. Int J Epidemiol 1994;23 (5):957-67.

34. Zmirou D, Barumandzadeh T, Balducci F, Ritter P, Laham G, Ghilardi J. Short term effects of air pollution on mortality in the city of Lyon, France, 1985-90. J Epidemiol Community Health 1996; 50 Suppl 1:S30-5.
35. Sunyer J, Castellsague J, Sáez M, Tobias A, Antó J. Air pollution and mortality in Barcelona. J Epidemiol Community Health 1996;50 Suppl 1: s 76 -s 80

36. Anderson H, Ponce de Leon A, Bland J, Bower J, Strachan D. Air pollution and daily mortality in London: 1987-92 [see comments]. BMJ 1996; 312(7032):665-9.

37. Ballester F, Corella D, Pérez Hoyos S, Hervás A. Air pollution and mortality in Valencia, Spain: a study using the APHEA methodology. J Epidemiol Community Health 1996;50:527-33.

38. Michelozzi P, Forastiere F, Fusco D, Penucci C, Ostro B, Ancona C et al. Air pollution and daily mortality in Rome, Italy. Occup Environ Med 1998;55:605-10.

39. Katsouyanni K. Health effects of air pollution in southern Europe: are there interacting factors? Environ Health Perspect 1995;103 Suppl $2: 23-7$.

40. Katsouyanni K, Touloumi G, Spix C, Schwartz J, Balducci F, Medina S et al. Short-term effects of ambient sulphur dioxide and particulate matter on mortality in 12 European cities: results from time series data from the $\triangle \mathrm{PHE} \Lambda$ project. Air Pollution and Health: a European Approach. BMJ $1997 ; 314(7095): 1658-63$.

41. Pitard $\Lambda$, Viel J. Some Methods to Address colli nearity among pollutants in epidemiological time serics. Stat Med 1997;16:527-44.

42. Forasticre F, Pcrucci C, Di Pietro A, Miccli M, Rapiti E, Bargagli A et al. Mortality among urban policemen in Rome. Am J Ind Med 1994;26 (6):785-98.

43. Janssen N, Hock G, Brunekreef B, Harssema H, Mensink I, Zuidhof A. Personal sampling of particles in adults: relation among personal, indoor, and outdoor air concentrations. Am J Epidemiol $1998 ; 147(6): 537-47$.

44. Le Tertre A, Quénel P, Medina S, Le Moullec Y, Festy $\mathrm{B}$, Ferry $\mathrm{R}$ et al. Modélisation des liens à court terme entre la pollution atmosphérique et la santé. Un exemple: $\mathrm{SO}_{2}$ el mortalité totale, Paris, 1987-1990. Rev Epidemiol Santé Publique 1998;46:316-28.

45. Katsouyanni K, Schwartz J, Spix C, Touloumi G, Zmirou D, Zanobetti A et al. Short term effects of air pollution on health: a European approach using epidemiologic time series data: the APHEA protocol. J Epidemiol Community Health 1996; 50 Suppl 1:S12-8. 